\title{
The differences in postural stability between cross-country and Alpine skiers with intellectual disabilities
}

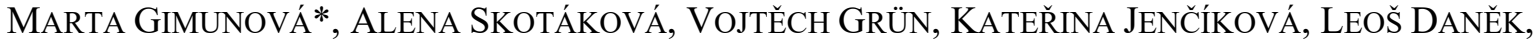 \\ TOMÁŠ HLINSKÝ, LENKA SVOBOdOVÁ, MiRiam KaliCHOVÁ, HaNA VÁlKOVÁ \\ Faculty of Sports Studies, Masaryk University, Brno, Czech Republic.
}

\begin{abstract}
Purpose: The purpose of this study was to analyse differences in postural stability between athletes with ID competing in Alpine and Cross country (XC) skiing to assess the effect of special sports training on postural stability in persons with ID. Methods: Athletes with ID were divided into four groups by their discipline: Alpine skiers $(n=9)$, XC skiers 2.5 to $10 \mathrm{~km}(n=10)$, XC skiers 500 to $1000 \mathrm{~m}(n$ $=18)$ and XC skiers 50 and $100 \mathrm{~m}(n=10)$. All participants were asked to stand barefooted on the Zebris FDM platform (Medical $\mathrm{GmbH}$, Germany) with their eyes open and closed. To compare differences between groups one-way ANOVA with Tukey HSD test was used. To compare the difference between the eyes open and closed, $t$-test was used. Results: Statistical analysis showed no significant differences between Alpine and XC skiers in analysed variables. The analysis of COP velocity showed superior postural stability in XC skiers 2.5 to $10 \mathrm{~km}$ compared to XC skiers 500 and $1000 \mathrm{~m}$. In XC skiers 500 and $1000 \mathrm{~m}$, XC skiers 2.5 to $10 \mathrm{~km}$ and Alpine skiers a significant increase in COP velocity was observed when having the eyes closed. In XC skiers 50 and $100 \mathrm{~m}$, no statistically significant differences between the eyes open and closed conditions were observed. Conclusions: Despite different sports training, no difference between Alpine and XC skiers in postural stability was observed. When comparing XC skiers, better postural stability was observed in skiers running longer distances, suggesting that this sports training enhances balance skills needed in everyday life.
\end{abstract}

Key words: intellectual disability, alpine skiing, cross-country skiing, Special Olympics, balance

\section{Introduction}

People with intellectual disability (ID) have increased risk of falling, compared to general population [1], [2]. In postural stability tests, persons with ID show poorer results compared to their peers without ID [3], [4]. In previous studies, physical activity was observed to have a positive effect on postural balance in persons with ID of various ages [6], [7].

The balance performance depends on the quality of CNS and motivation, and mood of the person being tested [8] and includes sensory information provided by vision, proprioception and vestibular system processed by the cerebellum, brainstem, basal ganglia and sensorimotor cortex [8], [9]. The CNS forms the appropriate muscle synergies needed to control the cen- ter of mass (COM) between the person's base of support and estimated by center of pressure (COP) measures. ID was observed to limit the motor development including balance skills [8], [10].

Alpine skiing requires fine postural control to maintain balance in challenging conditions. In a previous study by Noé and Paillard [12], professional Alpine skiers without ID were observed to perform inferior postural performance compared to lower level skiers when standing barefooted as the effect of ski boots repetitive wearing. On the other hand, a seven days long Alpine skiing training was reported to improve significantly the balance performance in beginners and intermediate skiers [13]. In a similar study involving a nine day long Alpine skiing training [14], the improvement in balance performance was observed only when wearing the skiing boots indicating the need for the

\footnotetext{
* Corresponding author: Marta Gimunová, Faculty of Sports Studies, Masaryk University, Brno, Czech Republic, Kamenice 5, 62500, Brno, Czech Republic. Phone: +420728367050, e-mail: gimunova@fsps.muni.cz

Received: June 30th, 2020

Accepted for publication: October 14th, 2020
} 
body to adapt to the restriction of the ankle joints mobility when wearing the ski boots.

In cross-counry skiing (XC skiing), the gliding phase is dependent on postural stability, and the postural instability was observed to cause a significant reduction in force output [15]. In a longitudinal study on elderly XC skiers, an improvement in balance performance was observed after six years despite the age of the participants [16]. A number of tools used for balance development during the pre-training and training in professional $\mathrm{XC}$ skiing has been discussed.

Special Olympics provides the competition and training for athletes with ID, under 75 points IQ. During the competition, athletes are grouped by age, gender and ability to provide all participant the chance to win. In Alpine skiing, the events differ from $10 \mathrm{~m}$ walk (gliding) to advanced slalom and Super-G. In XC skiing, official Special Olympics events differ from $10 \mathrm{~m}$ to $10 \mathrm{~km}$ races [18], [19].

The purpose of this study was to analyse the differences in postural stability between athletes with ID competing in Alpine skiing (intermediate and advanced slalom) and three categories of XC skiing (50 m to $10 \mathrm{~km}$ races) to assess the effect of specific training on postural stability in people with ID. Additionally, the differences in postural stability between female and male skiers were compared.

\section{Methods}

\section{Participants}

47 athletes with mild-to-moderate nonsyndromic ID, participating in Alpine or XC skiing races during the Winter Games of Czech Special Olympics Movement participated in this study. By their discipline they were divided into four groups: Alpine skiing (intermediate and advanced slalom, $n=9$ ), XC skiing 2.5 to $10 \mathrm{~km}$ race $(n=10)$, XC skiing 500 to $1000 \mathrm{~m}$ race $(n=18)$ and XC skiing 50 and $100 \mathrm{~m}$ race $(n=10)$. Their characteristics are described in Chapter 3. According to the XC skiing sport rules of Special Olympics, the coach is responsible for an appropriate selection of the race to the athlete skills. Athletes with lower fitness and/or more profound ID compete at shorter races in XC skiing. This ability grouping is giving everyone a reasonable chance to win [18]. The Word Winter Special Olympic Games started in 1977 and each discipline has its own special characteristics [5]. The Alpine skiing training begins with ski walking, straight run, Christie-type linked turns, and develop into open parallel turns [19]. The XC skiing 50 and $100 \mathrm{~m}$ requires classical technique with diagonal striding or double pole. $\mathrm{XC}$ skiing 500 and $1000 \mathrm{~m}$, and 2.5 to $10 \mathrm{~km}$ are free technique events where uphill (herringbone, side-step) and downhill (wedge, step turns) techniques are required as for events ranging from $1 \mathrm{~km}$ race the uphill, downhill, flat sections and sections with turns are recommended by the Special Olympic rules [18].

The inclusion criteria of this study consisted of no gait abnormalities, no lower limb injuries and more than 18 years of age. To compete within the Czech Special Olympics Movement the athlete has to attend regular trainings during the year, not only the competition (at least 60 minutes of physical activity per week). Informed consent was provided by all participants or their caregivers prior the screening. The screening was performed in accordance with ethical standards of the Helsinki Declaration.

\section{Procedures}

All participants were asked to stand upright, barefooted, arms along the body, both feet (shoulderwidth) on the Zebris FDM platform (Medical GmbH, Germany) for 20 seconds with their eyes open looking straight ahead and for 20 seconds with their eyes closed. The body height and body mass were measured using an anthropometer and personal scale (Tanita BC 601, Ltd., Paris, France), respectively. Additionally, participants (or their caregivers) provided information about their age and sport discipline. The screening was performed as a part of Healthy Athlete: Fit Feet program of Special Olympics by the Fit Feet clinical director and trained volunteers. Participation took place during the athlete's rest day (not during the day when they had the competition).

The following variables were analysed in both, eyes open and eyes closed conditions: 95\% confidence ellipse area (Ellipse area $\left[\mathrm{mm}^{2}\right]$ ), mean velocity of COP (COP velocity $[\mathrm{mm} / \mathrm{s}]$ ), relative distribution [\%] of force between the left (L) and right (R) foot (Total force L [\%], Total force R [\%]) and between the forefoot and backfoot (Forefoot force L [\%], Backfoot force L [\%], Forefoot force R [\%], Backfoot force R [\%]).

\section{Statistical analysis}

To compare the differences between the groups (Alpine skiers, XC skiers 2.5 to $10 \mathrm{~km}, \mathrm{XC}$ skiers 500 to $1000 \mathrm{~m}$, and $\mathrm{XC}$ skiers 50 and $100 \mathrm{~m}$ ), one-way ANOVA was used as the data showed a normal (Gaussian) distribution tested by Lilliefors test. The posthoc Tukey's HSD test was used to analyse the statistical differences between groups. To compare the difference between the eyes open and eyes closed condi- 
tion within the groups, paired samples $t$-test was used. To compare the differences between female and male participants, unpaired $t$-test was used. $P<0.05$ was considered to be statistically significant. The statistics were obtained using the Statistica Statsoft 12.

\section{Results}

The analysis of gender differences in postural stability variables showed no statistically significant differ-

Table 1. Results of $t$-test comparing differences between female and male participants

\begin{tabular}{|c|c|c|c|c|c|c|}
\hline & & \multicolumn{2}{|c|}{ Male $(n=28)$} & \multicolumn{2}{|c|}{ Female $(n=19)$} & \multirow[b]{2}{*}{$p$} \\
\hline & & Mean & SD & Mean & SD & \\
\hline \multirow{8}{*}{ Eyes open } & Ellipse area $\left[\mathrm{mm}^{2}\right]$ & 316.32 & 239.11 & 277.06 & 179.44 & 0.546 \\
\hline & COP velocity $[\mathrm{mm} / \mathrm{s}]$ & 9.83 & 4.34 & 9.32 & 4.16 & 0.695 \\
\hline & Forefoot force L [\%] & 50.47 & 5.49 & 50.32 & 7.50 & 0.935 \\
\hline & Backfoot force L [\%] & 49.53 & 5.49 & 49.68 & 7.50 & 0.935 \\
\hline & Total force L [\%] & 42.84 & 9.97 & 41.71 & 10.90 & 0.714 \\
\hline & Forefoot force R [\%] & 50.13 & 9.42 & 48.83 & 5.23 & 0.589 \\
\hline & Backfoot force R [\%] & 49.87 & 9.42 & 51.17 & 5.23 & 0.589 \\
\hline & Total force R [\%] & 57.16 & 9.97 & 58.29 & 10.90 & 0.714 \\
\hline \multirow{8}{*}{ Eyes closed } & Ellipse area $\left[\mathrm{mm}^{2}\right]$ & 297.87 & 231.04 & 360.86 & 303.80 & 0.424 \\
\hline & COP velocity $[\mathrm{mm} / \mathrm{s}]$ & 12.43 & 6.54 & 12.42 & 6.77 & 0.997 \\
\hline & Forefoot force L [\%] & 50.79 & 5.32 & 50.86 & 7.24 & 0.969 \\
\hline & Backfoot force L [\%] & 49.21 & 5.32 & 49.14 & 7.24 & 0.969 \\
\hline & Total force L [\%] & 44.19 & 8.54 & 42.41 & 10.80 & 0.531 \\
\hline & Forefoot force R [\%] & 49.37 & 10.08 & 49.37 & 7.90 & 0.997 \\
\hline & Backfoot force R [\%] & 50.63 & 10.08 & 50.63 & 7.90 & 0.997 \\
\hline & Total force R [\%] & 55.81 & 8.54 & 57.59 & 10.80 & 0.531 \\
\hline
\end{tabular}

Table 2. Mean and SD of analysed variables for all groups and results of ANOVA

\begin{tabular}{|c|c|c|c|c|c|c|c|c|c|c|}
\hline & & \multicolumn{2}{|c|}{$\begin{array}{l}\text { Alpine skiers } \\
\quad(n=9)\end{array}$} & \multicolumn{2}{|c|}{$\begin{array}{c}\text { XC skiers } \\
2.5 \text { to } 10 \mathrm{~km} \\
\quad(n=10)\end{array}$} & \multicolumn{2}{|c|}{$\begin{array}{c}\text { XC skiers } \\
500 \text { and } 1000 \mathrm{~m} \\
(n=18)\end{array}$} & \multicolumn{2}{|c|}{$\begin{array}{c}\text { XC skiers } \\
50 \text { and } 100 \mathrm{~m} \\
(n=10)\end{array}$} & \multirow[b]{2}{*}{$n$} \\
\hline & & Mean & SD & Mean & SD & Mean & SD & Mean & SD & \\
\hline \multirow{2}{*}{ Gender } & Female $(n)$ & \multicolumn{2}{|c|}{7} & \multicolumn{2}{|c|}{1} & \multicolumn{2}{|c|}{7} & \multicolumn{2}{|c|}{4} & \\
\hline & Male $(n)$ & \multicolumn{2}{|c|}{2} & \multicolumn{2}{|c|}{9} & \multicolumn{2}{|c|}{11} & \multicolumn{2}{|c|}{6} & \\
\hline \multicolumn{2}{|l|}{ Age [years] } & 33.56 & 9.40 & 37.00 & 7.70 & 32.89 & 11.60 & 44.70 & 16.18 & 0.080 \\
\hline \multicolumn{2}{|c|}{ Body height [cm] } & 166.06 & 9.06 & 173.65 & 5.33 & 168.56 & 8.15 & 165.70 & 8.11 & 0.106 \\
\hline \multicolumn{2}{|c|}{ Body mass [kg] } & 70.72 & 10.50 & 78.56 & 14.95 & 80.06 & 20.91 & 75.62 & 22.71 & 0.656 \\
\hline \multirow{8}{*}{ Eyes open } & Ellipse area $\left[\mathrm{mm}^{2}\right]$ & 290.71 & 169.36 & 173.65 & 121.70 & 373.62 & 267.99 & 304.30 & 182.23 & 0.133 \\
\hline & COP velocity $[\mathrm{mm} / \mathrm{s}]$ & 8.56 & 2.00 & 6.76 & 2.75 & 10.97 & 4.73 & 11.03 & 4.71 & $* 0.039^{\mathrm{A}}$ \\
\hline & Forefoot force L [\%] & 53.54 & 5.82 & 50.05 & 4.68 & 48.18 & 6.55 & 51.98 & 6.88 & 0.161 \\
\hline & Backfoot force L [\%] & 46.46 & 5.82 & 49.95 & 4.68 & 51.82 & 6.55 & 48.02 & 6.88 & 0.161 \\
\hline & Total force L [\%] & 39.09 & 11.09 & 44.80 & 10.47 & 43.55 & 10.04 & 40.84 & 10.28 & 0.598 \\
\hline & Forefoot force R [\%] & 49.47 & 3.84 & 50.97 & 8.89 & 49.63 & 9.03 & 48.31 & 8.55 & 0.911 \\
\hline & Backfoot force R [\%] & 50.53 & 3.84 & 49.03 & 8.89 & 50.37 & 9.03 & 51.69 & 8.55 & 0.911 \\
\hline & Total force R [\%] & 60.91 & 11.09 & 55.20 & 10.47 & 56.45 & 10.04 & 59.16 & 10.28 & 0.598 \\
\hline \multirow{8}{*}{ Eyes closed } & Ellipse area $\left[\mathrm{mm}^{2}\right]$ & 293.45 & 148.73 & 268.36 & 190.38 & 425.86 & 352.42 & 220.64 & 149.07 & 0.182 \\
\hline & COP velocity $[\mathrm{mm} / \mathrm{s}]$ & 11.06 & 2.82 & 10.32 & 4.85 & 15.19 & 8.46 & 10.79 & 5.43 & 0.154 \\
\hline & Forefoot force L [\%] & 54.21 & 5.01 & 49.73 & 5.05 & 49.57 & 6.79 & 51.11 & 6.23 & 0.279 \\
\hline & Backfoot force L [\%] & 45.79 & 5.01 & 50.27 & 5.05 & 50.43 & 6.79 & 48.89 & 6.23 & 0.279 \\
\hline & Total force L [\%] & 41.14 & 11.50 & 44.77 & 8.60 & 45.74 & 8.74 & 40.19 & 9.52 & 0.405 \\
\hline & Forefoot force R [\%] & 50.78 & 5.28 & 48.64 & 9.98 & 49.96 & 10.49 & 47.79 & 9.56 & 0.892 \\
\hline & Backfoot force R [\%] & 49.22 & 5.28 & 51.36 & 9.98 & 50.04 & 10.49 & 52.21 & 9.56 & 0.892 \\
\hline & Total force R [\%] & 58.86 & 11.50 & 55.23 & 8.60 & 54.26 & 8.74 & 59.81 & 9.52 & 0.405 \\
\hline
\end{tabular}

${ }^{\text {A }}$ Statistically significant difference found between XC skiers 2.5 to $10 \mathrm{~km}$ and XC skiers 500 and $1000 \mathrm{~m}$ groups.

* Highlights the statistical significance. 
Table 3. Results of $t$-test for all groups between the eyes open and eyes closed conditions

\begin{tabular}{|l|c|c|c|c|}
\hline & $\begin{array}{c}\text { Alpine } \\
\text { skiers }\end{array}$ & $\begin{array}{c}\text { XC skiers } \\
2.5 \text { to } 10 \mathrm{~km}\end{array}$ & $\begin{array}{c}\text { XC skiers } \\
500 \text { and } 1000 \mathrm{~m}\end{array}$ & $\begin{array}{c}\text { XC skiers } \\
50 \text { and } 100 \mathrm{~m}\end{array}$ \\
\cline { 2 - 5 } & $p$ & $p$ & $p$ & $p$ \\
\hline Ellipse area $\left[\mathrm{mm}^{2}\right]$ & 0.960 & $* 0.044$ & 0.492 & 0.206 \\
\hline COP velocity [mm/s] & $* 0.029$ & $* 0.006$ & $* 0.007$ & 0.843 \\
\hline Forefoot force L [\%] & 0.589 & 0.851 & 0.064 & 0.337 \\
\hline Backfoot force L [\%] & 0.589 & 0.851 & 0.064 & 0.337 \\
\hline Total force L [\%] & 0.054 & 0.986 & 0.119 & 0.674 \\
\hline Forefoot force R [\%] & 0.315 & 0.151 & 0.731 & 0.652 \\
\hline Backfoot force R [\%] & 0.315 & 0.151 & 0.731 & 0.652 \\
\hline Total force R $[\%]$ & 0.054 & 0.986 & 0.119 & 0.674 \\
\hline
\end{tabular}

* highlights the statistical significance.

ences between female and male participants (Table 1). Therefore, for the following analysis, female and male participants were analysed together.

The participants' characteristics, mean and SD of analysed variables and the results of ANOVA are shown in Table 2.

The one-way ANOVA and post-hoc Tukey's HSD test showed statistically significant difference only in COP velocity between the XC skiers 2.5 to $10 \mathrm{~km}$ and $\mathrm{XC}$ skiers 500 and $1000 \mathrm{~m}$ groups $(p=0.049)$. No statistically significant difference between the Alpine skiers and XC skiing groups was found.

In Table 3, the results of paired samples $t$-test comparing the analysed variables when having the eyes open and eyes closed are shown. The results show statistically significant differences between eyes open and closed conditions in Ellipse area in XC skiers 2.5 to $10 \mathrm{~km}$ group, and in COP velocity in Alpine skiers, XC skiers 2.5 to $10 \mathrm{~km}$ and XC skiers 500 and $1000 \mathrm{~m}$. In XC skiers 50 and $100 \mathrm{~m}$ group, no statistically significant differences between the eyes open and closed condition were observed.

\section{Discussion}

Despite a different sport training, no difference between Alpine and XC skiers with ID in postural stability variables was observed. The analysis of COP velocity showed the superior postural stability performances in $\mathrm{XC}$ skiers 2.5 to $10 \mathrm{~km}$ group when having the eyes open, compared to XC skiers 500 and $1000 \mathrm{~m}$. The COP velocity increased when having the eyes closed in both groups, similarly to the observation of Suomi and Koceja [21], showing no statistically significant difference in COP velocity between groups when having the eyes closed. The XC skiing was reported previously to improve balance [16] and the 2.5 to $10 \mathrm{~km}$ group was the most advanced XC skiing group participating in this study. XC skiing on longer distances $(2.5$ to $10 \mathrm{~km})$ seems to improve the postural stability of persons with ID. Furthermore, the XC skiing was observed to be a more effective activity for generating a high energy expenditure and oxygen uptake compared to Alpine skiing [23] and a decrease risk of hypertension and all-cause mortality was observed in a leisure-time XC skiers without ID in previous studies [24], [25]. XC skiing on longer distances may be, therefore, recommended for athletes with ID to improve their health and balance skills. In a previous study by Bibrowicz et al. [20], no statistical differences between Special Olympics volleyball players and controls without ID in postural stability was observed, showing that the regular training may significantly improve the postural stability of people with ID. In previous study on participants with Down syndrome [17], the reported COP velocity during the eyes open and eyes closed condition was 13.3 and $18.9 \mathrm{~mm} / \mathrm{s}$, and 10.6 and $14.5 \mathrm{~mm} / \mathrm{s}$ for the anterio-posterior and medio-lateral COP velocity, respectively. In ablebodied young adults, the observed anterio-posterior COP velocity was 11.9 and 14.0 for males and 14.0 and $15.1 \mathrm{~mm} / \mathrm{s}$ for females for the eyes open and closed condition, respectively. The observed mediolateral COP velocity was 8.0 and 9.3 for males and 9.4 and $10.2 \mathrm{~mm} / \mathrm{s}$ for females for the eyes open and closed condition, respectively [11]. In this study, the average COP velocity differed from 6.76 to 11.03 , and 10.32 to $15.19 \mathrm{~mm} / \mathrm{s}$ in the eyes open and closed condition, respectively, showing similar postural stability in the participants with non-syndromic ID compared to reported results of both participants with Down syndrome and healthy young adults [11], [17].

When comparing eyes open and closed conditions, results of previous studies are contradictory. 
Study by Dellavia et al. [4], focused on postural stability of Special Olympic athletes, found no significant differences between the eyes open and eyes closed conditions. Similar finding was observed in $\mathrm{XC}$ skiers 50 and $100 \mathrm{~m}$ group, whose motor and/or intellectual abilities are limited compared to other $\mathrm{XC}$ skiers or Alpine skiers with ID participating in this study. On the other hand, previous studies by Suomi and Koceja [21] and Bieć et al. [31] observed a significant increase in COP length and velocity in eyes closed compared to eyes open condition in people with ID. Similarly, in the XC skiers 500 and $1000 \mathrm{~m}, \mathrm{XC}$ skiers 2.5 to $10 \mathrm{~km}$ and in Alpine skiers a significant increase in COP velocity was observed when having the eyes closed. The contradictory results of previous studies may come from different groups of participants which were insufficient to cover the great variation within the population with ID [1]. Results of this study suggest that vision influence more the COP velocity in athletes with ID with better motor skills. Also, in professional athletes, the postural performance was reported to depend more on visual information than in lower level athletes [12], [22].

In previous studies, gender was observed to both, affect [26], [27] and not affect [28], [29] the postural stability. In this study, no effect of gender on postural stability variables was observed. Still, the limits of this study consist of a low number of participants and different number of women and man participating at each discipline as a result of different gender sports preferences and a lower number of women with ID participating in sports at higher level [30].

Future studies analysing the postural stability of athletes with ID practising different sports might show which sports training is more effective for improving balance needed in everyday life and enhancing the quality of life of persons with ID.

\section{Conclusions}

The analysis of postural stability showed no statistically significant differences between athletes with ID competing in Alpine and XC skiing. When comparing $\mathrm{XC}$ skiers, better postural stability was observed in skiers running longer distances, suggesting that this sport training may enhance balance skills needed in everyday life. When comparing the eyes open and eyes closed conditions, the results of this study suggest that vision restriction influence the COP velocity more in athletes with ID with better motor skills, as no difference between the eyes open and closed condition was observed in XC skiers 50 and $100 \mathrm{~m}$.

\section{Acknowledgements}

This study was a part of a project "Gait analysis in specific population groups (MUNI/A/1082/2019)" supported by Masaryk University, Brno, Czech Republic.

\section{References}

[1] Blomqvist S., Olsson J., Wallin L., Wester A., Rehn B., Adolescents with intellectual disability have reduced postural balance and muscle performance in trunk and lower limbs compared to peers without intellectual disability, Research in Developmental Disabilities, 2013, 34 (1), 198-206.

[2] Sherrard J., Tonge B.J., OzAnNe-Smith J., Injury in young people with intellectual disability: descriptive epidemiology, Injury Prevention, 2001, 7, 56-61.

[3] Carmeli E., Bar-Yossef T., Ariav C., Paz R., Sabbag H., LEVY R., Sensorimotor Impairments and Strategies in Adults with Intellectual Disabilities, Motor Control, 2008, 12 (4), 348-361.

[4] Dellavia C., Pallavera A., Orlando F., Sforza C., Postural Stability of Athletes in Special Olympics, Perceptual and Motor Skills, 2009, 108 (2), 608-622.

[5] VÁlKovÁ H., Winter sports within Special Olympics, Studia Sportiva, 2013, 7 (3), 291-305.

[6] Guidetti L., Franciosi E., Gallotta M.C., Emerenziani G.P., BALDARI C., Could sport specialization influence fitness and health of adults with mental retardation?, Research in Developmental Disabilities, 2010, 31 (5), 1070-1075.

[7] JANKOWICZ-SZYMAŃSKA A., MikOŁAJCZYK E., WOJTANOWSKI W., The effect of physical training on static balance in young people with intellectual disability, Research in Developmental Disabilities, 2012, 33 (2), 675-681.

[8] Kubilay N.S., Yildrim Y., Kara B., AKduR H.H., Effect of balance training and posture exercises on functional level in mental retardation, Fizyoterapi Rehabilitasyon, 2011, 22 (2), 55-64.

[9] Hatzitaki V., Zisi V., Kollias I., Perceptual-motor contributions to static and dynamic balance control in children, Journal of Motor Behavior, 2002, 34, 161-170.

[10] Klavina A., Jekabsone I., Static balance of persons with intellectual disabilities, visual impairment and without disabilities, European Journal in Adapted Physical Activity, 2004, 7 (1), 50-57.

[11] BŁaszczyk J.W., Beck M., Sadowska D., Assessment of postural stability in young healthy subjects based on directional features of posturographic data: Vision and gender effects, Acta Neurobiologiae Experimentalis, 2014, 74 (4), 433-442.

[12] NoÉ F., PAILlARD T., Is postural control affected by expertise in alpine skiing?, British Journal of Sports Medicine, 2005, 39, 835-837.

[13] Wojtyczek B., Pasławska M., Raschner C., Changes in the Balance Performance of Polish Recreational Skiers after Seven Days of Alpine Skiing, Journal of Human Kinetics, 2014, 44 (1), 29-40. 
[14] StanisZewski M., ZyBKo P., WisZOMIRSKA I., Influence of a nine-day alpine ski training programme on the postural stability of people with different levels of skills, Biomedical Human Kinetics, 2016, 8 (1), 24-31.

[15] Kurz A., Lauber B., Franke S., Leukel C., Balance Training Reduces Postural Sway and Improves Sport-specific Performance in Visually Impaired Cross-Country Skiers, Journal of Strength and Conditioning Research, 2018, DOI: 10.1519/JSC.0000000000002597.

[16] Lippens V., NAGel V., Balance performance of elderly cross-country skiers standing still or swaying smart?, [in:] E. Müller, S. Lindinger, T. Stöggl (Eds.), Science and Skiing $I V$, Meyer \& Meyer, Maidenhead, UK, 2009, 621-629.

[17] Bieć E., Zima J., WÓJTOWicz D., WOJCIEChOWSKA-MaszKowsKa B., KręCisz K., KuCZYŃSKi M., Postural stability in young adults with Down syndrome in challenging conditions, PLoS One, 2014, 9 (4), e94247.

[18] Special Olympics Cross Country Skiing Resources (2020). Version June 2020, Special Olympics, Inc. https://www. specialolympics.org/our-work/sports/cross-country-skiing

[19] Special Olympics Alpine Skiing Resources (2020). Version June 2020, Special Olympics, Inc. https://resources. specialolympics.org/sports-essentials/sports-and-coaching/alpineskiing?_ga=2.174201061.611577333.1591362953-268111195. 1525437154

[20] Bibrowicz K., Szurmik T., Wodarski P., Michnik R., Myśliwiec A., Barszcz J., Mikolajewski G., Mitas A., Quality of body posture and postural stability in people with intellectual disability playing volleyball, Acta of Bioengineering and Biomechanics, 2019, 21 (1), 23-30.

[21] SuOMi R., Koceja D.M., Postural sway patterns of normal men and women and men with mental retardation during a two-legged stance test, Archives of Physical Medicine and Rehabilitation, 1994, 75 (2), 205-209.

[22] Paillard T., Costes-Salon C., Lafont C., Dupui P., Are there differences in postural regulation according to the level of competition in judoists?, Br. J. Sports Med., 2002, 36 (4), 304-305.

[23] StöGgl T., Schwarzl C., Müller E.E., Nagasaki M., StÖGGl J., Scheiber P., SchÖNFElder, M., Niebauer J., A Comparison between Alpine Skiing, Cross-Country Skiing and Indoor Cycling on Cardiorespiratory and Metabolic Response, Journal of Sports Science and Medicine, 2016, 15 (1), 184-195.

[24] KunUtSOR S.K., MäKIKALlio T.H., KAUHANEN J., Voutilainen A., Jae, S.Y., KuRL, S., LAUKKANEN J.A., Leisure-time cross-country skiing is associated with lower incidence of hypertension, Journal of Hypertension, 2019, 37 (8), 1624-1632.

[25] LaukKanen J.A., LaukKanen T., Kunutsor S.K., Crosscountry skiing is associated with lower all-cause mortality: A population-based follow-up study, Scand. J. Med. Sci. Sports, 2018, 28, 1064-1072.

[26] Ku P.X., Abu Osman N.A., Yusof A., Wan Abas W.A.B., Biomechanical evaluation of the relationship between postural control and body mass index, Journal of Biomechanics, 2012, 45, 1638-1642.

[27] Sell T.C., LovaleKar M.T., Nagai T., WirT M.D., AbT J.P., LePHART S.M., Gender Differences in Static and Dynamic Postural Stability of Soldiers in the Army's 101st Airborne Division (Air Assault), Journal of Sport Rehabilitation, 2018, 27 (2), 126-131.

[28] Hageman P.A., Leibowitz J.M., Blanke D., Age and gender effects on postural control measures, Archives of Physical Medicine and Rehabilitation, 1995, 76 (10), 961-965.

[29] CruZ-Gómez N.S., Plascencia G., Villanueva-Padrón L.A., JÁUREGUI-RENAUD K., Influence of Obesity and Gender on the Postural Stability during Upright Stance, Obes. Facts, 2011, 4, 212-217.

[30] Van de Vliet P., Rintala P., Fröjd K., Verellen J., Van Houtte S., Daly D.J., VanlandewiJck Y.C., Physical fitness profile of elite athletes with intellectual disability, Scandinavian Journal of Medicine and Science in Sports, 2006, 16, 417-425. 